\title{
3 Community engagement in the informed consent process in global clinical research
}

\section{International recommendations and guidelines}

\author{
Margherita Daverio
}

\section{Challenges to informed consent in global clinical research and the need for community involvement}

Informed consent is not only a written form or a bureaucratic procedure but also, above all, an essential communication process between the participant and the researcher in clinical research. In many cases, obtaining informed consent may be difficult with people from diverse cultural backgrounds. It could happen in the context of global clinical research, particularly in the case of international multicentre studies where researchers and the potential participants belong to different cultural contexts. ${ }^{1}$

To overcome communication barriers and avoid misconceptions and misunderstandings, interaction in a multicultural setting cannot overlook cultural diversity, as it contributes to shaping subjective identities. Thus, cultural diversity affects the way people process and understand information.

Cultural differences between researchers and potential participants in clinical trials could result in communication barriers which are likely to hinder awareness and pose challenges to the informed consent process. ${ }^{2}$ In 2015, the World Health Organization (WHO) emphasized that "a challenge in global health ethics concerns international research, especially where investigators from wealthy countries conduct research in impoverished settings where participants are especially vulnerable or where language and cultural barriers make informed consent difficult." 3 In addition, in crosscultural communication - as in the case of specific international multicentre clinical trials in the context of global health-related research-special care is generally recommended in collecting informed consent to avoid the risk of possible poor communication due to language differences. ${ }^{4}$ Moreover, sound comprehension of information becomes complex when those who intervene do not use the same references in approaching health problems. For example, the scientific approach of a research team is different from a

DOI: $10.4324 / 9781003213215-5$ 
mystical, supernatural approach to health which could be found in some communities. ${ }^{5}$

We can identify as follows three major areas of barriers in cross-cultural communication:

a) language barriers; ${ }^{6}$ in some communities, there could not even be the word to express some scientific concepts related to research, for example, for the term "randomization"; 7 a study from the United Kingdom about the inclusion of non-English-speaking patients in the researchreported language barriers and the unavailability of translators for different reasons; ${ }^{8}$

b) lack of awareness about trials and shallow understanding of the concept of research, which may be confused with the direct health services provision; in general, difficulties in understanding research process; ${ }^{9}$

c) lack of trust in researchers and low health literacy regarding immunization; concern about adverse events and fears about exploitation (especially in the case of healthy volunteers, as it is in the case of experimental vaccines). ${ }^{10}$

A scientific paper highlighted several issues in the considered area (Kenya and sub-Saharan Africa) that undermined informed consent processes. ${ }^{11}$ These include limited exposure to research concepts and procedures, a lack of local terms for critical elements of research, rumours about research activities and their purposes, such as taking blood samples from healthy children. In addition, there could be difficulties for potential participants in understanding large volumes of research-related information, especially when children are sick and consenting processes are deemed to be delaying initiation of treatment. Finally, perceptions that research procedures are part of standard care (therapeutic misconceptions) or vice versa and lack of in-depth understanding of research or research ethics among those responsible for explaining research activities can hinder proper communication in the informed consent process. To respond to these challenges, researchers implemented informed consent forms prepared directly in the local language with the help of community members. ${ }^{12}$

Therefore, community engagement in research has gained consideration as an approach "helping ensure that community concerns are taken into account and to informing ethical decision-making when research is conducted in context of vulnerability." 13 During the whole informed consent process, community collaboration is essential to prevent researchers who are not local to a community from making assumptions about how to research and giving advice for conducting the process for seeking consent in a culturally tailored way. ${ }^{14}$ Relationships between communities and 
researchers can take different forms, ranging from community consultation in specific stages of the research to community representation during the whole research process and even to a long-term and more complex partnership. Different types of community involvement could be appropriate in different situations; for example, informal consultations could be enough for some studies, but in others, especially when vulnerable populations are involved, there could be a need for a formal consultation or partnership. ${ }^{15}$ In this perspective, community engagement becomes an essential part of research to meet each community's needs and priorities and overcome cultural challenges and communication barriers.

\section{Ethical goals of community engagement in collaborative research}

Community engagement is a recognized ethical requirement in healthrelated research. ${ }^{16}$ A community consists not only of people living in the geographic area where research is to be carried out, but it also comprises "different sectors of society that have a stake in the proposed research, as well as sub-populations from which research participants will be recruited."17 Engagement has ethical implications - ethics does not stop when community engagement takes place: 'Engagement is about 'exchange.' It is not about providing information or disseminating ideas or results. Communities can also drive the engagement process, holding scientists and science accountable for their ethics. Suppose a good relationship is already established between scientists and communities. In that case, it is possible to do a short piece of research that also engages the community as a matter of principle." 18 In addition, community engagement is a form of patient and public involvement (PPI). PPI in research can potentially help researchers make sure that their research design is relevant that is participant friendly and ethically sound. ${ }^{19}$ Community engagement could then be described as a double-way process, a mutual aid between the community and the research team and vice versa. ${ }^{20}$

To overcome the most frequent challenges to the informed consent process in the context of global health-related research, community engagement practices can promote a significant involvement of local people. Notably, community members should be invited to develop the informed consent process and documents to ensure that they are understandable and appropriate for potential participants. To face language barriers, the involvement of community members in the preparation of informed consent materials can help. For example, they can suggest how to explain to potential participants concepts that could be difficult to understand, such as "placebo," "randomization" and advising on how to provide information 
in a culturally sensitive way. ${ }^{21}$ To prevent from possible cultural barriers, potential cultural sensitivities should be explored in advance of biomedical research with the help of local communities. The contribution of potential local participants and local researchers can prevent violating customary practices. $^{22}$ This contribution often can come through the contribution of the local trusted "spokesperson," persons who not only can translate but also help understand cultural values and perceptions; one of these persons could be a contact person between the community and the research team.

Three main arguments supporting community engagement could be identified. ${ }^{23}$ They reflect the main ethical goals of community engagement:

1 Protecting communities. Informing and consulting members of the broader community on the ongoing research is seen as additional protection for the ethical conduct of the research besides that provided by ethics committee approvals and informed, ${ }^{24}$

2 Improving research quality. Community involvement has the potential to increase study efficiency in several steps: the better informed a community is about a study, the easier it will be to improve the recruitment process; research could benefit from a paradigm shift in which community involvement is seen as a way to achieve higher research quality; ${ }^{25}$

3 Building trust. Involving the community in the research is one way to build mutual trust with the population and show respect to all affected by the research, beyond the study participants. Building trust involves the commitment to the well-being of communities and the protection of their interests. ${ }^{26}$

Collaborative research views all stakeholders as equals, and even if the needs and priorities of the community and the researchers are not the same, they should be reciprocal. Increasingly, "international collaborative research is being asked to consider the local interests of resource-constrained partners and the responsibility of collaborations to safeguard against the potential for structural exploitation when operating in resource-constrained settings." ${ }^{27}$ Community engagement can set the basis for good collaborative research, which international recommendations and guidelines require, mainly through protecting communities, providing benefits for the community, research quality and building trust and equitable partnership among stakeholders. ${ }^{28}$

\section{Towards a meaningful participatory process. International recommendations and guidelines on community engagement}

Community engagement is central to any public health intervention. In 2017, the WHO emphasized its definition of community engagement as 
"a process of developing relationships that enable stakeholders to work together to address health-related issues and promote well-being to achieve positive health impact and outcomes." 29

In global clinical research, research can take place also in developing countries. Regarding this specific case, in 2003, the European Group on Ethics in Science and New Technologies (EGE) reminded that "the legitimacy of the objectives of research is related to the analysis of its relevance regarding health priorities of the partners, the risk/benefits balance for individuals and the communities, and the potential impact on healthcare of the host country." 30 To reach this ethical goal, researchers have an additional responsibility towards the community where the clinical trial takes place and should seek agreement as appropriate from people representative of or invested with certain authority in the community. Community engagement is a crucial means to tailor research and particularly informed consent process to the community needs and values. "The application of general ethical standards of clinical trials to the different cultural context, in particular to developing countries, needs in fact an activity of interpretation and specification: this process might be helped by a community consultation to acquire better knowledge of local culture and involving community representatives in the elaboration of research projects." ${ }^{\prime 1}$

The Council for International Organizations of Medical Sciences (CIOMS) guidelines in the revised 2016 version include a brand-new guideline devoted explicitly to community engagement. To this aim, Guideline 7 recommends the inclusion of communities in global collaborative research through a "meaningful participatory process, that involves them in an early and sustained manner in the design, development, implementation, design of the informed consent process and monitoring of the research, and in the dissemination of its results." 32

First, proactive and sustained engagement of communities from which individuals are invited to participate shows respect towards the communities and their traditions and norms. ${ }^{33}$ Second, besides successful conduct of research, community engagement is a means of ensuring the relevance of proposed research to the affected community and ensures the ethical and social value and outcome of the proposed research. Third, community engagement is a double-way ongoing process, which promotes reciprocal trust and confidence between researchers and community members. "Active engagement with community members is a mutually educative process, which both enables researchers to learn about communities' cultures and understanding of research-related concepts and contributes to research literacy by educating the community about key concepts critical for understanding the purpose and procedures of the research." ${ }^{34}$

Community engagement implies mutual and reciprocal relationships between the research team and community members. In addition, CIOMS 
guideline 7 reminds that engaging the community strengthens local ownership of the research and builds confidence in the ability of leaders to negotiate various aspects of the research (recruitment strategies, care for the health needs of study participants, site selection, data collection and sharing and so on).

Engagement is recommended, "at the earliest opportunity." Engaging at the earliest stage promotes smooth study functioning. It contributes to the community's capacity to understand the research process - and by design, as research protocol to be submitted to the research ethics committee should include a detailed description of the plan for community engagement. Although strategies could be different, community engagement should have an established forum for communication between researchers and community members.

According to CIOMS Guideline 8, Collaborative partnership and capacity building for research and research review, local capacity building is identified as an ethical aspect. The collaborative partnership helps ensure the social value of research by engaging the communities, thereby focusing on aspects the community considers valuable. The act of entering a partnership in collaborative research has accompanying ethical responsibilities. This marks the need for collaborative research partnerships to contribute to sustainable capacity-building activities that bring structured changes to local skills, knowledge and systems. ${ }^{35}$

Community's permission under no circumstance can substitute individual informed consent. ${ }^{36}$ Community consent cannot substitute individual informed consent according to international ethical requirements. ${ }^{37}$ On the issue of community consent, it has been emphasized that recognizing the potential for community protection offered by deliberately reflexive ethical review boards, including community consultations, would avoid the problematic notion of "community consent," which on the other side would imply that "communities" can consent to ethical, legally valid and socially meaningful research. ${ }^{38}$ As an effective strategy, "both forms of protectionsindividual informed consent and ethical review of research protocolsshould work together in promoting, strengthening, and reinforcing the protection of communities and their individual members involved in biomedical research." 39

\section{Leading strategies for community involvement in global clinical research}

As considered in the previous paragraph, community engagement is an ongoing and continuing process. Specific strategies and approaches should be tailored to the community context. ${ }^{40}$ 
In this perspective, choosing the most appropriate form of engagement is a delicate commitment. The best methods of engagement are those which are chosen by the community. ${ }^{41} \mathrm{~A}$ general principle is that to achieve successful collaboration with a community, all parties involved need to strive to understand the point of view of "insiders" of the community. In addition to ample information about community engagement, best practices and case studies, the report called "Principles of community engagement" provides nine community engagement principles, which help identify community engagement strategies. ${ }^{42}$ Among these principles are researchers should be clear with the community about purposes and goals of engagement; researchers should know as much as possible the local culture they will be going to interact with; direct interaction with members of the community should be promoted; research teams should foster partnerships and collaborative research; researchers should have and show respect for cultural values and belief and the community self-determination; capacity building should be implemented; flexibility should be promoted to meet the community changing needs and research teams should be available to long-term commitment in building trust.

An active community engagement is, therefore, recommended, and strategies and practical advice could be identified as follows: ${ }^{43}$

a) the inclusion of members of the community involved in the clinical trial in the Institutional Review Board, to include community views and perspectives in the ethical review of the study; ${ }^{44}$

b) to build long-term, mutually beneficial relationships between the community and the research team, to be applied before, during and after research studies with different strategies according to different phases of the research study, for example: engaging in full and frank discussions about the potential benefits and harms that the participants and the community might encounter; ensuring the alignment of the research to local needs; paying the due attention to the impact of the study and the study team upon the participants, their families, the local community and the environment; taking the time to ensure that the implications of the study have been fully understood by participants and the community; ${ }^{45}$

c) the consultation with community members, ${ }^{46}$ in particular on how to work with the community, for example, providing a forum for discussing and addressing issues arising from participants and community representatives ${ }^{47}$ an ongoing "dialogue" between the researchers and the community about the proposed study and its potential implications, or a more structured consultation that would document the concerns of a community or a socially identifiable group; 
d) periodical meetings with local health teams to inform them of potential studies, including meeting in the local languages with the key leaders in the community, including the village head, the religious leaders; community feedback meetings at the end of the trials also proved helpful in informing participants that studies had ended and for sharing study results; ${ }^{48}$ collaborative efforts revealed insights about how to convey information about clinical trial participation;

e) In disease outbreaks, community engagement should be reached through social media, ${ }^{49}$ particularly as far as preparedness and risk communication is concerned. ${ }^{50}$

To reach community engagement ethical goals, researchers need to be specifically trained. ${ }^{51}$

\section{Could community engagement be a step more towards a relational view of autonomy?}

Community engagement in the informed consent process in global clinical research recognizes and considers the value of relational and cultural dimensions of everyone. Community engagement practices respect these dimensions and drive their contributions into the decision-making process, fostering the exercise of autonomy without undermining it.

We suggest that community engagement practices and the involvement of community members in the informed consent process could be thought of as a way of moving towards a relational view of autonomy. This view can be achieved considering the need for community involvement in the research process and decision-making. Accordingly, they consider the cultural and relational dimensions of everyone as described in the previous paragraphsnever overcoming personal autonomy, always protecting and safeguarding individual and free decision-making. This is because the challenge of respecting the autonomy of persons is not to avoid relational dimensions but to include meaningful forms of relationship in the decision-making process. ${ }^{52}$

A growing number of bioethicists and social scientists invoke a "relational turn" in bioethics that moves beyond individualistic towards more relational perspectives..$^{53}$ They are sketching a reconfiguration of the principle of autonomy. A relational view of autonomy includes the autonomy of the individual and the reference to the dialogue with the researcher/physician and wife/husband/relatives and members of the community. ${ }^{54}$ This approach can provide solutions to ethical and practical problems in clinical practice and research.

A "relational turn" of autonomy should not be confused with collectivism or authoritarian and paternalistic versions of communitarianism. ${ }^{55} \mathrm{In}$ 
clinical research and the informed consent process, individual informed consent should not be overwhelmed by community consent, as stated in international recommendations and above-recalled guidelines. However, concerning the autonomy principle, clear emphasis should be devoted to the situated and communal aspects of relational life and interdependence. A person's interests are connected to other people and groups. They could, thus, be read as a call for a degree of relational autonomy. "Not independence, but interdependence, is at the heart of relational notions of autonomy: social surroundings and relationships enable us to flourish and develop a robust capacity for self-determination and identity formation. Relational autonomy can be viewed as a conception of autonomy that places the individual in a socially embedded network of others. Relationships (with family, community, and society), responsibility, care and interdependence are key attributes of relational autonomy: people develop their sense of self and form capacities and life plans through the relationships they forge on a daily and long-term basis. Therefore, relational autonomy asserts that social surroundings and relationships are crucial for developing autonomy and encourages us to act in ways guided by an ethic of trust and care." ${ }^{56}$

In addition, relational autonomy can provide a broader moral framework for the informed consent process, as the individual is, indeed, autonomous, but at the same time, community-oriented and not self-sufficient and independent of others. According to relational autonomy, informed consent can be a process and a non-static concept. In the case of community engagement practices, the process of consultation gives substantive weight to the considerations of relationships, patients as relational beings who are socially embedded..$^{57}$ Therefore, a relational concept of autonomy is a precious concept to appeal to in biomedical research and clinical practice to protect rights and dignity.

In this perspective, community engagement intended as a "meaningful participatory process," involving communities, considering relational dimensions, respecting cultural values and always safeguarding personal autonomy could be inserted in the ethical framework of a relational view of autonomy. This view could be deepened, indeed, more than in the space of this chapter.

\section{Notes}

1 Henk Ten Have and Bert Gordijn (eds.), Handbook of Global Bioethics (Cham, Switzerland: Springer, 2013), 154.

2 i-CONSENT Project, Ethical and Legal Review of Gender and Age-Related Issues Associated with the Acquisition of Informed Consent, i-CONSENT Project (2017), https://i-consentproject.eu/wp-content/uploads/2019/02/D1.3_EthicalLegal_ 20171030_FINAL.pdf; Doris Schroeder, et al., Ethics Dumping Case Studies 
from North-South Research Collaborations (New York: Springer, 2018), 99-106; i-CONSENT Project, New Strategies for Increasing Participation of Patients Coming from Different Cultural and Religious Backgrounds (2019), www.lumsa. it/sites/default/files/link/T2.6\%20LUMSA\%20summary\%20of\%20findings April\%2026\%202019_final.pdf.

3 World Health Organization, Global Health Ethics: Key Issues (2015), https:// apps.who.int/iris/bitstream/handle/10665/164576/9789240694033_eng.pdf;jses sionid=77B9CE5DDD86423B93D0BF4868F76E82? sequence=1.

4 UNESCO International Bioethics Committee, Report of the IBC on Consent (2008), https://unesdoc.unesco.org/ark:/48223/pf0000178124; The Council of Europe, Guide for Research Ethics Committee Members, Steering Committee on Bioethics (2012), www.coe.int/t/dg3/healthbioethic/activities/02_biomedical research_en/Guide/Guide_EN.pdf; Council for International Organizations of Medical Sciences (CIOMS), International Ethical Guidelines for Health-Related Research Involving Humans, Geneva (2016), https://cioms.ch/wp-content/ uploads/2017/01/WEB-CIOMS-EthicalGuidelines.pdf, accessed May 17, 2021.

5 UNESCO International Bioethics Committee, Report of the IBC on Consent, 106.

6 Schroeder, et al., Ethics Dumping Case Studies from North-South Research Collaborations, 99-106; Louise Condon, et al., "Engaging Gypsy, Roma, and Traveller Communities in Research: Maximizing Opportunities and Overcoming Challenges," Qualitative Health Research 29, no. 9 (2019): 1324-1333, https:// doi.org/10.1177/1049732318813558; Laura Palazzani, Innovation in Scientific Research and Emerging Technologies: A Challenge to Ethics and Law (Cham, Switzerland: Springer Nature and G. Giappichelli Editore, 2019).

7 George Okello, et al., "Challenges for Consent and Community Engagement in the Conduct of Cluster Randomized Trial among School Children in Low Income Settings: Experiences from Kenya," Trials 14, no. 1 (2013): 142. https:// doi.org/10.1186/1745-6215-14-142.

8 Rachel Bernier, et al., "Inclusion of Non-English-Speaking Patients in Research: A Single Institution Experience," Pediatric Anesthesia 28, no. 5 (2018): 415420. https://doi.org/10.1111/pan.13363.

9 Teo Quay, et al., "Barriers and Facilitators to Recruitment of South Asians to Health Research: a Scoping Review," BMJ Open 7, no. 5 (2017). https://doi. org/10.1136/bmjopen-2016-014889; Rossybelle P. Amorrortu, et al., "Recruitment of Racial and Ethnic Minorities to Clinical Trials Conducted within Specialty Clinics: An Intervention Mapping approach," Trials 19, no. 1 (2018): 1-10. https://doi.org/10.1186/s13063-018-2507-9.

10 Amorrortu, et al., "Recruitment of Racial and Ethnic Minorities"; Joyce L. Browne, et al., "The Willingness to Participate in Biomedical Research Involving Human Beings in Low- and Middle-Income Countries: A Systematic Review," Tropical Medicine \& International Health 24, no. 3 (2019): 264-279. https://doi. org/10.1111/tmi.13195; Sarah Gehlert and Jessica Mozersky, "Seeing Beyond the Margins: Challenges to Informed Inclusion of Vulnerable Populations in Research," Journal of Law, Medicine \& Ethics 46, no. 1 (2018): 30-43. https:// doi.org/10.1177/1073110518766006; Bernadette Boden-Albala, et al., "Examining Barriers and Practices to Recruitment and Retention in Stroke Clinical Trials," Stroke 46, no. 8(2015): 2232-2237. https://doi.org/10.1161/strokeaha.114.008564.

11 Mwanamvua Boga, et al., "Strengthening the Informed Consent Process in International Health Research through Community Engagement: The KEMRI-Wellcome Trust Research Programme Experience," PLoS Medicine 8, no. 9 (2011). https://doi.org/10.1371/journal.pmed.1001089. 
12 Boga, et al., "Strengthening the Informed Consent Process."

13 Kathleen M. MacQueen, et al., "Evaluating Community Engagement in Global Health Research: The Need for Metrics," B.M.C. Medical Ethics, 16, no. 44 (2015): 1-10.

14 Emily F. Fitzpatrick, et al., "Seeking Consent for Research with Indigenous Communities: A Systematic Review," BMC Medical Ethics 17, no. 1 (2016). https://doi.org/10.1186/s12910-016-0139-8.

15 Federica Fregonese, "Community Involvement in Biomedical Research Conducted in the Global Health Context: What Can Be Done to Make It Really Matter?," BMC Medical Ethics 19, no. S1 (2018). https://doi.org/10.1186/ s12910-018-0283-4.

16 CIOMS, International Ethical Guidelines; European Group on Ethics in Science and New Technologies, Ethical Aspects of Clinical Research in Developing Countries: Opinion (2003), n. 17, https://publications.europa.eu/en/publicationdetail/-/publication/6339dcbf-c156-4e7f-9e43-9928acf82118/language-en/ format-PDF/source-77404483.

17 CIOMS, International Ethical Guidelines, Commentary on Guideline 7.

18 Wellcome Trust, Community Engagement:-Under the Microscope: Report of a Workshop in Thailand (June 12-15, 2011), https://wellcome.ac.uk/sites/default/ files/wtvm054326_0.pdf.

19 Heather J. Bagley, Hannah Short, Nicola L. Harman, Helen R. Hickey, Carrol L. Gamble, Kerry Woolfall, Bridget Young, and Paula R. Williamson, "A Patient and Public Involvement (PPI) Toolkit for Meaningful and Flexible Involvement in Clinical Trials:-A Work in Progress," Research Involvement and Engagement 2, no. 1 (2016). https://doi.org/10.1186/s40900-016-0029-8.

20 See CIOMS, International Ethical Guidelines; Vibian Angwenyi, "Complex Realities: Community Engagement for a Paediatric Randomized Controlled Malaria Vaccine Trial in Kilifi, Kenya," Trials 15, no. 65 (2014): 1-16. https:// doi.org/10.1186/1745-6215-15-65; Kate Chatfield, et al., "Research with, Not about, Communities:-Ethical Guidance towards Empowerment in Collaborative Research, a Report for the TRUST Project," TRUST Project (July 15, 2018), http://rust-project.eu/wp-content/uploads/2018/07/TRUST-CommunityParticipation-in-Research-Final.pdf.

21 Okello, et al., "Challenges for Consent and Community Engagement."

22 See TRUST Project, Global Code of Conduct for Research in Resource-Poor Settings (2018), www.globalcodeofconduct.org/wp-content/uploads/2018/05/ Global-Code-of-Conduct-Brochure.pdf, art. 8, "Respect."

23 Fregonese, "Community Involvement in Biomedical Research."

24 See also Patricia Marshall and Jessica W. Berg, "Protecting Communities in Biomedical Research," The American Journal of Bioethics 6, no. 3 (2006): 28-30. https://doi.org/10.1080/15265160600685770; MacQueen, et al., "Evaluating Community Engagement."

25 Fregonese, "Community Involvement in Biomedical Research."

26 Paulina Tindana, et al., "Grand Challenges in Global Health: Community Engagement in Research in Developing Countries," PLoS Medicine 4, no. 9 (2007). https://doi.org/10.1371/journal.pmed.0040273; MacQueen, et al., "Evaluating Community Engagement."

27 Claire Leonie Ward, et al., "Good Collaborative Practice: Reforming Capacity Building Governance of International Health Research Partnerships," Globalization and Health 14, no. 1 (2018). https://doi.org/10.1186/s12992-017-0319-4.

28 CIOMS, International Ethical Guidelines. 
29 World Health Organization, Community Engagement Framework for Quality, People-Centred and Resilient Health Services (Geneva: World Health Organization, 2017), https://apps.who.int/iris/bitstream/handle/10665/259280/WHOHIS-SDS-2017.15-eng.pdf.

30 European Group on Ethics in Science and New Technologies, Ethical Aspects of Clinical Research, par. 1.25.

31 Palazzani, Innovation in Scientific Research.

32 CIOMS, International Ethical Guidelines, Guideline 7.

33 Johannes J. van Delden and Rieke van der Graaf, "Revised CIOMS International Ethical Guidelines for Health-Related Research Involving Humans," JAMA 317 , no. 2 (2017): 135. https://doi.org/10.1001/jama.2016.18977.

34 CIOMS, International Ethical Guidelines, Commentary on Guideline 7.

35 Ward, "Good Collaborative Practice."

36 CIOMS, International Ethical Guidelines, Commentary on Guideline 9.

37 See World Medical Association, Declaration of Helsinki (1964), last version 2013, www.wma.net/policies-post/wma-declaration-of-helsinki-ethical-principlesfor-medical-research-involving-human-subjects/, art. 25, and UNESCO, Universal Declaration on Bioethics and Human Rights (2005), http://portal.unesco. org/en/ev.php-URL_ID=31058\&URL_DO=DO_TOPIC\&URL_SECTION= 201.html, art. 6.3.

38 Marshall and Berg, "Protecting Communities in Biomedical Research."

39 Ibid.

40 National Institutes of Health (NIH) - Clinical and Translational Science Awards Consortium, Community Engagement Key Function Committee, Task Force on the Principles of Community Engagement, Principles of Community Engagement, 2nd ed. (2011), www.atsdr.cdc.gov/communityengagement/pdf/PCE_ Report_508_FINAL.pdf.

41 Wellcome Trust, Community Engagement.

$42 \mathrm{NIH}$, Principles of Community Engagement.

43 Browne, "The Willingness to Participate in Biomedical Research."

44 European Medicines Agency, Reflection Paper on Ethical and GCP Aspects of Clinical Trials of Medicinal Products for Human Use Conducted in Third Countries and Submitted in Marketing Authorisation Applications to the E.M.A. (2010), www.ema.europa.eu/en/documents/regulatory-procedural-guideline/draftreflection-paper-ethical-good-clinical-practice-aspects-clinical-trials-medicinalproducts_en.pdf; CIOMS, International Ethical Guidelines.

45 Chatfield et al., "Research with, Not about, Communities."

46 Patricia Marshall, "Ethical Challenges in Study Design and Informed Consent for Health Research in Resource-Poor Settings," UNICEF/UNDP/World Bank/ WHO Special Programme for Research and Training in Tropical Diseases \& World Health Organization, https://apps.who.int/iris/bitstream/handle/10665/4 3622/9789241563383_eng.pdf;sequence=1, accessed May 17, 2021; CIOMS, International Ethical Guidelines.

47 Angwenyi, "Complex Realities."

48 Olubukola T. Idoko, et al., "Community Perspectives Associated with the African PsA-TT (MenAfriVac) Vaccine Trials," Clinical Infectious Diseases 61, no. suppl. 5 (2015). https://doi.org/10.1093/cid/civ596.

49 National Institute for Health and Care Excellence (NICE), Community Engagement: Improving Health and Well-Being and Reducing Health Inequalities (2016), http://nice.org.uk/guidance/ng44. 
50 World Health Organization, Risk Communication and Community Engagement Preparedness and Readiness Framework: Ebola Response in the Democratic Republic of Congo in North Kivu (2018), www.who.int/iris/handle/10665/ 275389, where a helpful checklist is provided.

51 Syed Ahmed and Ann-Gel Palermo, "Community Engagement in Research: Frameworks for Education and Peer Review," American Journal of Public Health 100, no. 8 (2010): 1380-1387 provide several educational strategies for research team about community engagement - usually traditional doctoral and master's programs do not train specifically on these aspects during researchers' education.

52 Bruce Jennings, "Reconceptualizing Autonomy: A Relational Turn in Bioethics," Hastings Center Report, 46 (2016): 11-16.

53 Jennings, "Reconceptualizing Autonomy"; Edward S. Dove, et al., "Beyond Individualism: Is There a Place for Relational Autonomy in Clinical Practice and Research?," Clinical Ethics 12, no. 3 (2017): 150-165. https://doi.org/ 10.1177/1477750917704156; Palazzani, Innovation in Scientific Research; Federico De Montalvo Jääskeläinen, Menores De Edad y Consentimiento Informado (Valencia: Tirant Lo Blanc, 2019); Silvina Alvarez Medina, La autonomía de las personas. Una capacidad relacional (Madrid: Centro de Estudios Políticos y Constitucionales, 2018).

54 The Italian Committee for Bioethics, Opinion on Migration and Health (2017), http://bioetica.governo.it/en/opinions/opinions-responses/migration-and-health/.

55 Jennings, "Reconceptualizing Autonomy."

56 Dove, et al., "Beyond Individualism."

57 See Peter I. Osuji, "Relational Autonomy in Informed Consent (RAIC) as an Ethics of Care Approach to the Concept of Informed Consent," Medicine, Health Care and Philosophy 21, no. 1 (2017): 101-111. https://doi.org/10.1007/s11019017-9789-7, where a relational view of autonomy in the informed consent process is grounded on an ethics of care. 